\title{
Defect Characteristics of Be-doped GaSb Film Grown on GaAs
}

\author{
Xin Guo ${ }^{1}$ and Yan Chen ${ }^{2, *}$ \\ ${ }^{1}$ School of Mechanical \& Electrical Engineering, Mianyang Teachers' College (Mianyang Normal University), Mianyang 621000 \\ China \\ ${ }^{2}$ School of Mathematics and Physics, Mianyang Teachers' College (Mianyang Normal University), Mianyang 621000 China \\ ${ }^{*}$ Corresponding author
}

\begin{abstract}
The defect characteristics of the Be-doped GaSb and no-doped GaSb film grown on GaAs and GaSb substrate respectively were analysed by the method of molecular beam epitaxy (MBE), positron annihilation Doppler broadening spectroscopy (PADBS), X-ray diffraction spectra (XRD) and atomic force microscopy (AFM). The experimental results show that the defects in Be-doped semiconductor GaSb could be attributed to existence of intrinsic defect, which has no complex defects in it. After doped Be atom, the crystallization of GaSb become worse, and the Be existed mainly in the interstitial atom.
\end{abstract} defect

Keywords-GaSb; positron annihilation spectroscop; XRD;

\section{INTRODUCTION}

Semiconductor $\mathrm{GaSb}$ has the unique band-structure alignment, small electron effective mass and high electron mobility [1-3]. Compared to the GaSb, GaAs can form excellent $n$ and $p$ Ohmic contacts, and has semi-insulating and favorable thermal properties. GaSb film based on GaAs usually has been applied to fabricate photoelectrical device [4]. The structure of film has recently attracted significant research interest because of its importance for photoelectrical performance. The p-type Si-doped, p-type Zi-doped, and n-type Te-doped of GaSb film have been published [5-7]. The p-type Be-doped of GaSb film has never been reported.

Many measurement techniques have been used to characterize the structure of the film, such as transmission electron microscopy (TEM), Rutherford backscattering (RBS), elastic recoil detection (ERD) analysis, X-ray diffraction (XRD) and positron annihilation spectroscopy (PAS) and so on [6]. Among them, the positron annihilation spectroscopy being easily susceptible to open-volume defects, it has been usually employed to probe the microscopic information of defects, such as their size, charge states, concentration, and chemical surrounding. The structure characteristics in GaSb films by variable energy positron annihilation spectroscopy (VEPAS) have been first carried out [7].

At present, doped-Be atom GaSb epitaxial layers grown on semi-insulating GaAs (001) substrates, another non-doped $\mathrm{GaSb}$ film grown on GaSb substrate with nearly same growth conditions. The atomic force microscope (AFM), XRD and Positron annihilation doppler broadening spectroscopy (PADB) were used to measurement it.

The full width at half maximum (FWHM) of the X-ray diffraction patterns of $\mathrm{GaSb}$ and Be-doped GaSb epitaxial layers were used to study, and analysis the trends of Doppler broadening S, W parameters of samples as well.

\section{EXPERIMENTAL PROCESS}

The Be-doped GaSb epitaxial layers grown on the top of GaAs (001) substrate, and the thickness about $580 \mathrm{~nm}$, the concentration of $\mathrm{Be}$ atom approximates to $10^{18} \mathrm{ppm}$ [8]. For comparative, choose another sample, which was non-doped GaSb film grown on GaSb substrate with nearly same conditions. The diffractometer of XRD (DX-1000) using $\mathrm{Cu}$ Ka X-ray source worked in a $2 \theta$ mode, scanning from $10^{\circ}$ to $80^{\circ}$ with a step of $0.06^{\circ}$, and the wavelength $\lambda$ is $1.5406 \AA$, the diffractometer operating voltage is $35 \mathrm{KV}$, accelerated current is $25 \mathrm{~mA}$. A slow positron beam was employed for positron doppler broadening measurements, which were carried out in a vacuum chamber at about $10^{-6} \mathrm{~Pa}$. The energy of the positron beam varied from 0.1 to $20 \mathrm{keV}$. The energy of $\gamma$ photo from the $\mathrm{e}^{+}-\mathrm{e}^{-}$annihilation radiation was recorded by a p-type high-purity germanium detector [9].

\section{RESULTS AND DISCUSSION}

Figure I (a-b) shows the AFM image of samples measured within the $10 \times 10 \mu \mathrm{m}$. From the AFM image can be seen, the sample A surface is smooth, no obvious spiral structure of dislocations and the island, indicating its has good crystallization. Figure II (a-b) shows the X-ray diffraction pattern of the 200 direction for $\mathrm{GaSb}$ and Be-doped $\mathrm{GaSb}$ epitaxial layers respectively. Referring to the Table I, another important point in this figure of the crystal in equality is that the FWHM, using the following relation [9]:

$$
F W H M=0.9 \lambda / t \cos \theta
$$
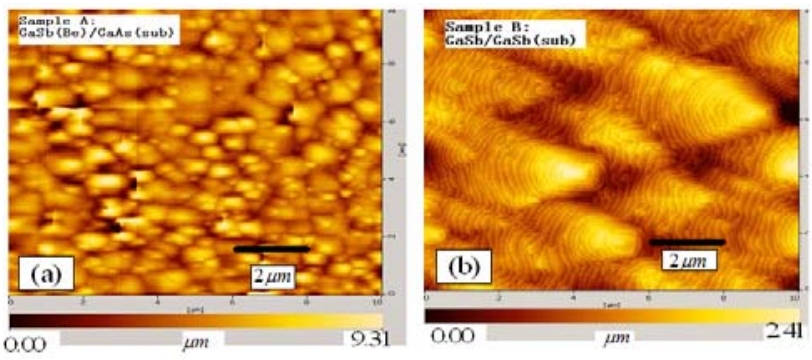

FIGURE I. THE PLAN-VIEW BRIGHT-FIELD AFM IMAGES OF SAMPLE A AND SAMPLE B. 

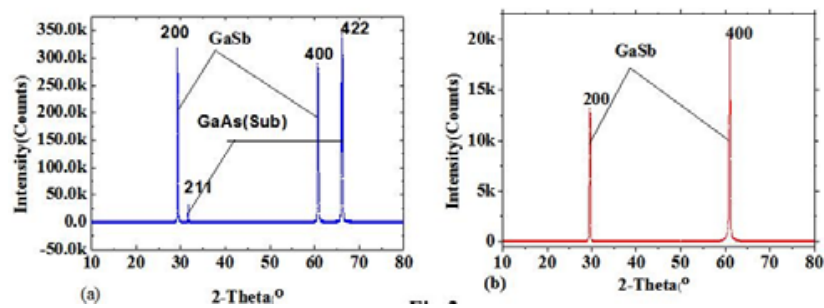

FIGURE II. THE X-RAY DIFFRACTION SPECTRA OF SAMPLE A AND SAMPLE B.

where $\mathrm{t}$ is size of crystallites, $\lambda$ is the wavelength of $\mathrm{Cu}-\mathrm{K}_{\alpha 1}$ radiation and $\theta$ is the Bragg's angle [10-14]. Result indicates that the Be-doped $\mathrm{GaSb}$ has better crystalline than the undoped $\mathrm{GaSb}$. Feel free to the GaSb which grown by molecular beam epitaxy (MBE) on GaAs substrates with high quality buffer layer has nearly $7 \%$ lattice mismatch between the GaAs and $\mathrm{GaSb}$ [8]. Owing to the high level of Be, the crystallization is slightly well [15]

TABLE I. ANALYSIS RESULT OF X-RAY DIFFRACTION SPECTRA.

\begin{tabular}{llllll}
\hline \multicolumn{2}{l}{ Peaks of XRD } & GaSb 200 & GaAs 211 & $\begin{array}{l}\text { GaSb } \\
400\end{array}$ & $\begin{array}{l}\text { GaAs } \\
422\end{array}$ \\
\hline $\begin{array}{l}\text { FWHM of } \\
\text { sample A }\end{array}$ & $0.141^{\circ}$ & $0.126^{\circ}$ & $0.156^{\circ}$ & $0.191^{\circ}$ \\
$\begin{array}{l}\text { FWHM } \\
\text { sample B }\end{array}$ & $0.265^{\circ}$ & & $0.325^{\circ}$ & \\
\hline
\end{tabular}

The cure of trends of the positron annihilation Doppler broadening S-parameters and $\mathrm{W}$-parameters are shown in Figure III (a-b). From above curve, the S-parameter and $\mathrm{W}$-parameters as a function of different energies has a significant variation with depth of near the epitaxial layers and the substrate. The mean implantation depth of the positron is calculated using following equation [17]:

$$
H=40 E^{1.6} / \rho^{40}
$$

Where $\mathrm{Z}$ is expressed in units of nanometers, $\rho$ is the density in units of $\mathrm{g} / \mathrm{cm}^{3}$, and $\mathrm{E}$ is the incident energy in kiloelectronvolts.
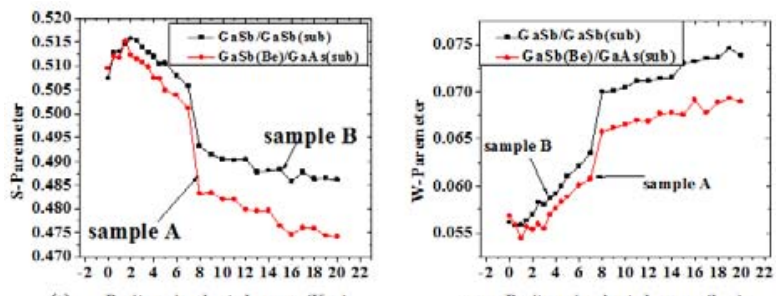

FIGURE III. THE TREND OF DOPPLER BROADENING S-PAREMETERS(A) AND W-PAREMETERS(B).

In Figure III (a), at $8 \mathrm{KeV}$ the slop of curve fast increasing means that energy which below near $8 \mathrm{KeV}$ injected to epitaxial layers and above near $8 \mathrm{KeV}$ injected to the substrate [18-21]. Calculated the S-parameters of sample A were smaller than sample B, this result indicated that quality of sample $A$ is better than sample $B$, in other words, the crystallization doped $\mathrm{Be}$ is better than non-doped in epitaxial layers and in substrate, and this was good agreement with the result of measurement of

\section{XRD and AFM.}

However, compared to the S-parameters of sample A and sample B in epitaxial layers and in substrate respectively, as we can see, the gap of S-parameters is even smaller in epitaxial layers than that in substrate. It is possible that the number and size of new micro-defects increase after doped $\mathrm{Be}$, and the crystallization slightly becomes worse than non-doped. After doped the high level of $\mathrm{Be}$, it seems that the inclusion of substitutional Be led to the crystallization slightly becomes worse.

The value of W-parameters indicated that there were more annihilating positron electrons with high momentum. The electron momentum information at different depth was represented by the values of $\mathrm{W}$-parameters as well. The cure of trends of the positron annihilation Doppler broadening $\mathrm{W}$-parameters are shown in Figure III (b). The W-parameters of sample A was less than sample B by calculated. This result indicated that the crystallization doped Be slightly become worse than non-doped, and this was good agreement with the result of the S-parameters.

Analysis the S-W parameter, we can observe the positron interface states owing to the positron annihilation in the interface has the unique information. From the Figure IV. curve, shows the variation of Doppler broadening S-W parameters within from $1 \mathrm{KeV}$ to $8 \mathrm{KeV}$. Having no large mutations in the curve of sample, and almost located in the same straight line, it seems to be there were no complex defects in it [22].

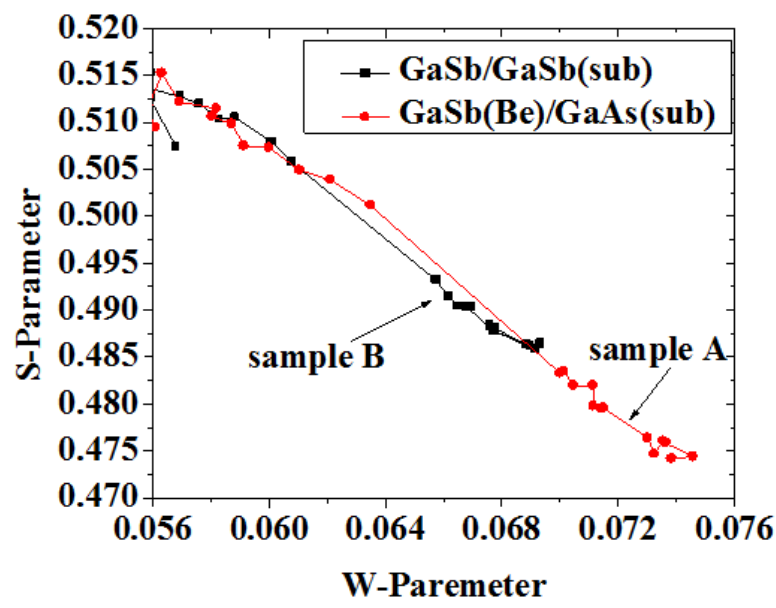

FIGURE IV. THE TREND OF DOPPLER BROADENING S-W PARAMETERS.

\section{CONCLUSIONS}

In conclusion, the crystallization of Be-doped $\mathrm{GaSb}$ film slightly became worse than that of non-doped sample. In GaSb, $V_{s b}^{0}$ occurs at doping levels near the top of the valence band with formation energy of $2.73 \mathrm{eV}$, and $V_{G a}^{0}$ has formation energy of $1.79 \mathrm{eV}$ as well, $V_{G a}^{p}$ accordingly dominate and are likely to have much higher concentrations than $V_{s b}^{p}$ [23]. The PADBS demonstrated that the crystallization of Be-doped sample become worse than that of non-doped sample. The high concentration of $\mathrm{Be}$ atom formed the inclusion of substitution, 
accordingly, some vacancies of $\mathrm{Sb}$ decrease as well, and $\mathrm{Be}$ doped in GaSb became p-type semiconductor [14], and the type of defects in the Be-doped p-type semiconductor $\mathrm{GaSb}$ could be attributed to the existence of interstitial atom.

\section{ACKNOWLEDGEMENTS}

This work was financially supported by the National Natural Science Foundations of Mianyang Teachers' College (Nos MYSY2017JC10).

\section{REFERENCES}

[1] C. Mourad, D. Gianardi, R. Kaspi, $2^{\mu} \mathrm{m}$ GaInAsSb/AlGaAsSb midinfrared laser grown digitally on $\mathrm{GaSb}$ by modulated-molecular beam epitaxy, J. Appl. Phys. 88 (2000) 5543-5546.

[2] A. Chattopadhyay, K. K. Das, Electronic Spectroscopy of the AlSb Molecule: A Theoretical Study, J. Phys. Chem. A 107 (2003) 6047-6054.

[3] J.W. Luo, A. Franceschetti, A. Zunger, Carrier Multiplication in Semiconductor Nanocrystals: Theoretical Screening of Candidate Materials Based on Band-Structure Effects, Nano Lett. 8 (2008) 3174-3181.

[4] S.H. Huang, G. Balakrishnan, A. Khoshakhlagh, A. Jallipalli, L. R. Dawson, D. L. Huffaker, Strain relief by periodic misfit arrays for low defect density GaSb on GaAs, Appl. Phys. Lett. 88 (2006) 131911-131911-3

[5] J. Vincenta, C. Diaz-Guerra, J. Piqueras, A. Amariei, E.K.Polychroniadis, E. Dieguez, Characterization of undoped and Te-doped GaSb crystals grown by the vertical feeding method, J. Cryst. Growth 289 (2006) 18-23.

[6] C. C. Ling, S. Fung, C. D. Beling, Defect study of Zn-doped p-type gallium antimonide using positron lifetime spectroscopy, Phys. Rev B 64 (2001), 075201-075201-7

[7] S. Abroug, F. Saadallah, F. Genty, N. Yacoubi, Investigation of Electrical and Optothermal Properties of Si-doped GaSb epitaxial layers by the Hall Effect, PL measurement and Photothermal Deflection Spectroscopy, Phys. Procedia 2 (2009) 787-795.

[8] J. Guo, Z.Y. Peng, W.G. Sun, Y.Q. Xu, Z.Q. Zhou, Z.C. Niu, InAs/GaSb superlattices for photodetection in short wavelength infrared range,Infrared. Phys. Technol. 52 (2009) 124-126.

[9] L.R.Zhang. A.H. Deng, D.X. Yang, Y.L. Zhou, Q. HOU, L.Q. Shi, Y.R. Zhong, and B.Y. Wang, Bias Effects on the Growth of Helium-Containing Titanium Films, Chin. Phys. Lett. 28 (2011) 077802-077802-4.

[10] A. Rogalski, Infrared detectors: status and trends, Progress in Quantum Electronics 27 (2003) 59-210.

[11] Y.b. Li, Y. Zhang, Y.W. Zhang, B.Q. Wang, Z.P. Zhu, Y.P. Zeng, Molecular beam epitaxial growth and characterization of $\mathrm{GaSb}$ layers on GaAs $\left(\begin{array}{ll}0 & 0\end{array}\right)$ substrates, Appl. Surf. Sci. 258 (2012) 6571-6575.

[12] Smita Jha, C.C. Liu, T. S. Kuan, S. E. Babcock, P. F. Nealey, J. H. Park,L, J. Mawst, T. F. Kuech, Defect reduction in epitaxial GaSb grown on nanopatterned GaAs substrates using full wafer block copolymer lithography, Appl. Phys. Lett. 95 (2009) 062104-062104-3.

[13] S. Abroug, F. Saadallah, N. Yacoubi, Photothermal investigations of doping effects on opto-thermal properties of bulk GaSb, J. Alloys. Compounds 484 (2009) 772-776

[14] Y. Li, A.H. Deng, Y.L. Zhou, B. Zhou, K. Wang, Q. Hou, L.Q. Shi, X.B. Qin, B.Y. WANG, Helium-Related Defect Evolution in Titanium Films by Slow Positron Beam Analysis, Chin. Phys. Lett. 29 (2012) 047801-047801-4

[15] Vidya Jadhav, S.K. Dubey, R.L. Dubey, A.D. Yadav, D. Kanjilal, Structural and optical studies of GaSb implanted with iron ions, Surf Coat. Technol. 203 (2009) 2670-2673.

[16] A. Seeger, Positron diffusion in solids and in liquid metals, Appl. Surf. Sci. 85 (1995) 8-16.

[17] M. Zhao, J.D. Wang, D.R. Chen, X.P.Hao, B.Y. Wang, Positron annihilation study of the micro-defects induced by cavitation in mild
steel,Physica B 403 (2008) 2594-2596.

[18] Y.Y. Shan, A. H. Deng, C. C. Ling, S. Fung, C. D. Ling, Y. W. Zhao, T. N. Sun, N. F. Sun, Positron-annihilation study of compensation defects in InP, J. Appl. Phys. 91 (2002) 1998-2001.

[19] Y.D Shao, Z. Wang, Y.Q. Dai, Y.W. Zhao, F.Y. Tang, Identification of vacancies in electron irradiated $\mathrm{GaSb}$ by coincidence Doppler broadening spectroscopy, Mater. Lett. 61 (2007) 1187-1189.

[20] W.G. Hu, Z. Wang, B.F. Sua, Y.Q. Dai, Gallium antisite defect and residual acceptors in undoped GaSb, Phys Lett. A 332 (2004) 286-290.

[21] E.J. Koerperick, L.M. Murray, D.T. Norton, T.F. Boggess, J.P. Prineas, Optimization of MBE-grown GaSb buffer layers and surface effects of antimony stabilization flux, J. Cryst. Growth 312 (2010) 185-191.

[22] G.P. Karwasz, A. Zecca, R.S. Brusa, D. Pliszk, Application of positron annihilation techniques for semiconductor studies, J.Alloys. Compounds 382 (2004) 244-251.

[23] H.A. Tahini, A. Chroneos, S. T. Murphy, U. Schwingenschlögl, R. W. Grimes, Vacancies and defect levels in III-V semiconductors, J. Appl.Phys. 114 (2013) 063517-063517-9. 